\title{
Estrategias para resolver problemas matemáticos con ideas de Pólya, en grado quinto
}

\author{
Sandra Raquel Fonseca Cano* \\ Consuelo Jiménez Patiño*** \\ Miguel Patarroyo Mesa ${ }^{* * * *}$
}

Artículo de reflexión.

Fecha de Recepción: 8 diciembre 2017.

Fecha de Aprobación: 20 abril 2018.

\section{Resumen}

La finalidad del escrito que a continuación se presenta, es comunicar los resultados parciales de una investigación que se desarrolló en el marco del programa Becas para la Excelencia Educativa del Ministerio de Educación Nacional en convenio con la UPTC. La investigación tuvo como objetivo favorecer el aprendizaje matemático en la competencia de resolución de problemas de los estudiantes de grado quinto de las Instituciones Educativas Gustavo Rojas Pinilla (de la ciudad de Tunja) y Concha Medina de Silva (del municipio de Muzo), mediante la implementación de una propuesta pedagógica. Se desarrolló teniendo en cuenta el enfoque cualitativo, el tipo de investigación utilizado fue acción educativa; su implementación se hizo en tres momentos, diagnóstico mediante una prueba pre-test; con base en los resultados del diagnóstico, se diseñaron y aplicaron talleres; y se finaliza con una prueba post-test a manera de evaluación. A manera de conclusión, en la resolución de problemas de aplicación matemática, los estudiantes incorporan algunas estrategias para comprender con facilidad una situación problema, utilizan esquemas y representaciones distintas a la original y con la aplicación
* Institución Educativa Gustavo Rojas Pinilla, Boyacá (Colombia) sandraf395@gmail.com ** Institución Educativa Concha Medina de Silva, Boyacá (Colombia) consujipadaso@gmail.com **** Universidad Pedagógica y Tecnológica de Colombia, Boyacá (Colombia) miguel.patarroyo@uptc. edu.co

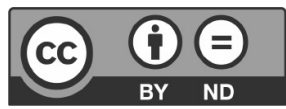


de la propuesta pedagógica, se genera acorde con la edad de los estudiantes y un ambiente propicio, atrayente, que el plan de estudios.

capta y cautiva la atención de los estudiantes, máxime cuando se apoya en material didáctico fácil de elaborar,

Palabras clave: análisis matemático, educación, matemática, investigación pedagógica, resolución de problemas. 


\section{Introducción}

El propósito de la investigación reside en una propuesta pedagógica en el área de matemáticas para los estudiantes de grado quinto de las Instituciones Educativas Gustavo Rojas Pinilla, de la ciudad de Tunja; y Concha Medina de Silva, del municipio de Muzo (departamento de Boyacá). El trabajo aborda la necesidad de superar las dificultades de los estudiantes para resolver problemas de aplicación matemática, situación reflejada en resultados de las pruebas Saber y, por ende, en el Índice Sintético de la Calidad Educativa (ISCE) y los informes del estado por institución, lo cual se complementó con el diagnóstico realizado, mediante el pretest, al inicio del trabajo. La importancia de proceder en torno a estas temáticas, que afectan a los estudiantes en los resultados de las diferentes pruebas aplicadas, y que redunda en el rendimiento académico de los alumnos, es la dificultad que presentan en la medida en que avanzan hacia otros niveles de educación, incluyendo la superior, es lo que dificulta o imposibilita su ingreso y permanencia en estas instituciones.

La investigación presenta un sustento referencial desde lo internacional, lo nacional y lo local. En el ámbito internacional investigaciones, que hayan hecho uso del método Pólya (1984); para la resolución de problemas, están: Escalante (2015); Villagrán y Navarro (2000); Calvo (2007); Fernández (2006); Romero (2011); en ellos, se observa que los estudiantes son capaces de resolver mecánicamente las operaciones fundamentales básicas, pero no saben cómo aplicarlas para la solución de un problema, ya que solo se les enseña a actuar de forma mecánica y repetitiva. Por ello, es fundamental tomar conciencia acerca de la problemática vivida en torno a este tema, y a su vez tomar las medidas necesarias para lograr el mejoramiento en los procesos de enseñanza y aprendizaje de la resolución de problemas.

En el mismo sentido, en el contexto nacional se encuentran trabajos como los de Agudelo, Bedoya y Restrepo (2008); Cortés y Galindo (2006); Murillo (2013), quienes basan sus investigaciones en el método heurístico de George Pólya (1984), e infieren que permite mejorar la capacidad resolutiva de problemas matemáticos en los estudiantes. Finalmente, en el entorno boyacense se encuentra a Barajas (2013), quien realiza su trabajo a partir de talleres para ser desarrollados en forma individual; luego, en grupos; y, por último, en plenaria, cada grupo socializa los resultados, argumentándolos y defendiéndolos.

Los trabajos investigativos mencionados, aportan al desarrollo del proceso en variados aspectos, la metodología, los referentes teóricos, el método, vienen a ser los que estructurarán y guiarán la investigación de forma adecuada y concreta.

De otro lado, la razón que conduce a diseñar e implementar una propuesta pedagógica a partir de las heurísticas plateadas por George Pólya (1984), es contribuir y fortalecer el desempeño
La investigación presenta un sustento referencial desde lo internacional, lo nacional y lo local. 
en la resolución de problemas en los estudiantes de grado quinto de las mencionadas instituciones; por tal motivo, se indagan estrategias que pueden ser utilizadas por los sujetos para resolver situaciones problema donde esté inmersa la aplicación matemática.

Diseñar, aplicar y evaluar una propuesta pedagógica que favorezca la resolución de problemas de aplicación matemática, es el objetivo de la investigación; esto es posible si se logra identificar el nivel y capacidad que tienen los estudiantes en cuanto a la interpretación y resolución de problemas matemáticos; así en el curso, diseñar y aplicar una propuesta pedagógica basada en el método de Pólya, que promueva el desempeño de los estudiantes en resolver situaciones con problema de aplicación matemática, y de esta manera finalizar el proceso investigativo comparando los resultados obtenidos mediante una valoración post-test para verificar si la propuesta pedagógica planteada es o no viable.

Respecto al tema central de la presente investigación, se encuentra, entre otros: la resolución de problemas, a través de la cual se incluyen diversos aspectos relacionados con la simbolización, representación, aplicación de reglas generales, traducción de unos lenguajes a otros, donde el aprendizaje de las matemáticas exige, en primer lugar, el dominio de códigos lingüísticos especializados; y, en segundo lugar, la capacidad de traducción desde otros códigos a los códigos matemáticos y viceversa. Uno de los problemas fundamentales consiste en que el alumno debe aprender a sustituir los procedimientos intuitivos y los códigos propios del lenguaje natural $\mathrm{u}$ ordinario por los procedimientos formales y códigos propios del lenguaje matemático.

De la misma manera, es relevante utilizar el método de resolución de problemas de George Pólya (comprender el problema, elaborar un plan, ejecutar el plan, revisión y verificación de la solución) como un proceso donde los estudiantes pueden analizar y reflexionar, permitiéndole mejorar la habilidad para resolver diferentes situaciones problema, superando actitudes que se han acentuado a través de los años, donde al alumno solo le interesa leer el texto dado y obtener una respuesta de forma rápida; mientras que, con los argumentos anteriores, se fortalece la competencia de resolución de problemas y se propician condiciones para ser utilizadas en otras áreas de estudio y en la vida cotidiana.

Lo anteriormente expuesto, constituye un proceso confuso de enseñanza que, en muchos casos, la escuela no promueve. Las dificultades de traducción no se producen solo entre la acción y la simbolización, sino también entre esta y el lenguaje verbal. En este caso, la traducción entre el lenguaje natural y el matemático no es directa. Es por ello que, es preciso analizar el texto, estableciendo la relación entre los datos con los que se cuenta, el orden en que aparecen y cómo se pueden utilizar para llegar a la 
solución, lo cual sobrepasa los límites de la simple comprensión del lenguaje utilizado .

Sin lugar a dudas, otro tema que apoya la investigación y que es particular en el ámbito escolar, es el referido a los términos "ejercicio" y "problema" empleados con singular frecuencia. Según Borasi (citada en Blanco, 1991), constituyen "ejercicios" (p. 35) aquellas tareas que pretenden desarrollar algún tipo de algoritmo. En consecuencia, para los ejercicios, el alumno tiene ya disponibles respuestas satisfactorias para las que ha sido preparado, y al contrario de lo que sucede en un verdadero problema: no hay incertidumbre en su comportamiento.

El trabajo con ejercicios no solo constituye el medio fundamental para la realización de los objetivos de la enseñanza de la Matemática, sino también el instrumento adecuado para la medición del rendimiento de los estudiantes. El éxito de la enseñanza de la Matemática no solo depende de cuáles ejercicios se plantean, sino también de cómo el profesor dirige su proceso de resolución. Respecto al término, problema matemático, Llivina precisa cuándo un ejercicio tiene carácter de problema, y expresa que: "Un ejercicio es un problema si y sólo si la vía de solución es desconocida para la persona" (Llivina, 1999, p. 48). Es decir, un ejercicio es problema cuando faltan los conocimientos específicos sobre el dominio de métodos o algoritmos de solución.

\section{Materiales y métodos}

En cuanto a la metodología utilizada, se trata de una investigación cualitativa de tipo investigación acción. Para el enfoque metodológico y el tipo de investigación, se acudió a Hernández \& Fernández (1998), quienes afirman que "La investigación cualitativa proporciona profundidad a los datos, dispersión, riqueza interpretativa, contextualización del ambiente, detalles y experiencias únicas. También aporta un punto de vista fresco, natural y completo de los fenómenos, así como flexibilidad" (p. 19); a Elliot (2000), quien define la investigación acción como un estudio de una situación social con el fin de mejorar la calidad de acción dentro de la misma, con el propósito de profundizar la comprensión del profesor (diagnóstico) de su problema; y a Kemmis \& MacTaggart (1988), para quienes la investigación acción está orientada hacia el cambio educativo y se caracteriza entre otras cosas por ser un proceso que se construyen desde y para la práctica.

La población objeto de estudio son los estudiantes de las Instituciones Educativas Gustavo Rojas pinilla y Concha Medina de Silva. La muestra seleccionada está constituida por 31 estudiantes de grado quinto de básica primaria y 22 de la Institución Educativa Gustavo Rojas Pinilla, son niños provenientes de vereda la Esperanza del municipio de Tunja, cuyas edades oscilan entre los nueve y once años, estrato socioeconómico $1 \mathrm{y}$ 2. El comportamiento social
Sin lugar a dudas, otro tema que apoya la investigación y que es particular en el ámbito escolar, es el referido a los términos "ejercicio" y "problema" empleados con singular frecuencia. 
en general es bueno, la actividad económica que predomina en la región es la explotación de minas de carbón. Además, 9 estudiantes de la Institución Educativa Concha Medina de Silva, las edades de los niños oscilan entre 9 y 13 años, estrato socioeconómico 1, la mayoría de las familias son disfuncionales, la actividad económica de los habitantes de la región es la extracción de esmeraldas.

De igual manera, la propuesta se trabaja, en primer lugar, con la aplicación del pretest, el cual inquiere las debilidades de los alumnos, presenta cinco situaciones problema de matemáticas, que los estudiantes desarrollan de manera habitual. Durante la aplicación, se evidencia que intentan comprender el problema mediante una lectura rápida y enseguida lo resuelven usando solo operaciones aritméticas fundamentales (suma, resta, multiplicación y división) que, en varios casos, no corresponden. En segundo lugar, se implementó la estrategia mediante el desarrollo de cinco talleres basados en el método de Pólya, en los cuales se presentan situaciones con problema de aplicación matemática con un nivel de dificultad bajo, explicando al inicio que van a resolver una situación teniendo en cuenta cuatro etapas: la primera es la etapa de comprensión, donde el estudiante hace una lectura de la situación, luego a nivel grupal y finalmente guiada por la docente; enseguida, se identifican las palabras desconocidas y buscan su significado, se elabora un plan, se aplica el mismo $\mathrm{y}$, por último, se hace una revisión $\mathrm{y}$ solución del problema; en el tercer momento, se evalúo la implementación de la propuesta mediante un post test (ex post) y comparando los resultados obtenidos, para obtener conclusiones respecto a los problemas planteados inicialmente y respondiendo al interrogante general desglosado a través de los objetivos.

\section{Análisis y discusión de resultados}

El proceso de análisis y discusión de los resultados se realiza en tres etapas, una parte para el diagnóstico, es decir, el análisis del pretest; otra consiste en la implementación de los talleres; y se finaliza con el análisis del postest. Finalmente, se hace la comparación y se establecen las conclusiones.

Para el análisis y discusión de los resultados, se tiene en cuenta la siguiente matriz de análisis de Resolución de problemas de aplicación matemática. En ella, aparecen categorías y subcategorías, así como las etapas del método Pólya, que para el caso son las categorías de análisis emergente (ver tabla 1).

En las subcategorías se encuentran, entre otros, criterios que permiten analizar y valorar características y condiciones que presentan los estudiantes en pre y postest frente a las etapas descritas por Pólya. 


\section{Tabla 1}

\section{Matriz de análisis}

\begin{tabular}{|c|c|c|c|c|}
\hline \multicolumn{5}{|c|}{ Matriz de Análisis } \\
\hline \multicolumn{5}{|c|}{ Resolución de problemas de aplicación matemática } \\
\hline Categorías & Etapa de comprensión & $\begin{array}{c}\text { Etapa de } \\
\text { configuración del plan }\end{array}$ & $\begin{array}{l}\text { Etapa de ejecución del } \\
\text { plan }\end{array}$ & Etapa de revisión y verificación \\
\hline \multirow{6}{*}{ Subcategorías } & $\begin{array}{l}\text { Lee detenidamente la } \\
\text { situación }(\mathrm{A})\end{array}$ & $\begin{array}{l}\text { Puede utilizar un } \\
\text { método empleado con } \\
\text { anterioridad (A) }\end{array}$ & $\begin{array}{l}\text { Establece plan de } \\
\text { solución }(\mathrm{A})\end{array}$ & $\begin{array}{l}\text { Son correctos los pasos y } \\
\text { operaciones (A) }\end{array}$ \\
\hline & $\begin{array}{c}\text { Determina si conoce el } \\
\text { significado de todas las } \\
\text { palabras }(\mathrm{B})\end{array}$ & $\begin{array}{c}\text { Agrega nuevos } \\
\text { elementos para } \\
\text { utilizar un método (B) }\end{array}$ & $\begin{array}{l}\text { Comprueba los pasos ya } \\
\text { realizados }(\mathrm{B})\end{array}$ & $\begin{array}{l}\text { Establece con precisión cuál fue } \\
\text { el paso clave en la solución (B) }\end{array}$ \\
\hline & $\begin{array}{l}\text { Determina qué datos dan } \\
\text { y qué piden }(\mathrm{C})\end{array}$ & $\begin{array}{l}\text { Son necesarios todos } \\
\text { los datos }(\mathrm{C})\end{array}$ & $\begin{array}{l}\text { Explica lo que hace y } \\
\text { para qué lo hace }(C)\end{array}$ & $\begin{array}{c}\text { Colabora a sus compañeros } \\
\text { para que desarrollen } \\
\text { habilidades en la solución de } \\
\text { situaciones }(\mathrm{C})\end{array}$ \\
\hline & $\begin{array}{l}\text { Dibuja y/o representa la } \\
\text { situación (D) }\end{array}$ & $\begin{array}{l}\text { Usa diferentes } \\
\text { estrategias (D) }\end{array}$ & $\begin{array}{l}\text { Soluciona el problema } \\
\text { completamente (D) }\end{array}$ & $\begin{array}{l}\text { Utiliza el resultado obtenido } \\
\text { para formular y plantear nuevas } \\
\text { situaciones (D) }\end{array}$ \\
\hline & $\begin{array}{l}\text { Encuentra relación entre } \\
\text { los datos y las incógnitas } \\
\text { (E) }\end{array}$ & $\begin{array}{l}\text { Revisa su lista de } \\
\text { estrategias (E) }\end{array}$ & $\begin{array}{l}\text { Reordena las ideas y } \\
\text { prueba de nuevo (E) }\end{array}$ & Se autoevalúa (E) \\
\hline & $\begin{array}{c}\text { Analiza el problema } \\
\text { desde varios ángulos }(\mathrm{F})\end{array}$ & $\begin{array}{l}\text { Recuerda si ha visto } \\
\text { una situación parecida } \\
\text { (F) }\end{array}$ & & $\begin{array}{l}\text { Lee nuevamente el enunciado y } \\
\text { comprueba }(\mathrm{F})\end{array}$ \\
\hline
\end{tabular}

Fuente: elaboración propia.

\section{Fase Diagnóstica: PreTest}

Situación 1: Sebastián tiene en su panadería, dos cajas. A y B con panes debidamente empacados en bolsas. En la caja $\mathrm{A}$, tiene bolsas con igual cantidad de panes cada una y no sobra ningún pan. En la caja B, tiene bolsas con igual cantidad de panes cada una y tampoco le sobran panes. El número de panes que hay en la caja A es igual al número de panes que hay en la caja $B$. ¿Cuántos panes hay en cada caja? 
Tabla 2

Etapa de comprensión y utilización de subcategorías

\begin{tabular}{|c|c|c|c|}
\hline \multicolumn{4}{|c|}{ Resultados de los estudiantes en la etapa de comprensión } \\
\hline & & Pre-test & Pos-test \\
\hline Institución educativa & Subcategoría & $\begin{array}{l}\text { Estudiantes } \\
\text { que } \\
\text { implementaron } \\
\text { cada categoría }\end{array}$ & $\begin{array}{l}\text { Estudiantes que } \\
\text { implementaron cada } \\
\text { categoría }\end{array}$ \\
\hline \multirow{6}{*}{$\begin{array}{l}\text { Gustavo Rojas } \\
\text { Pinilla } \\
22 \text { estudiantes }\end{array}$} & Lee detenidamente la situación. & 5 & 20 \\
\hline & $\begin{array}{l}\text { Determina si conoce el significado } \\
\text { de todas las palabras. }\end{array}$ & 0 & 18 \\
\hline & $\begin{array}{l}\text { Determina qué datos dan y qué } \\
\text { piden. }\end{array}$ & 5 & 20 \\
\hline & Dibuja y/o representa la situación. & 3 & 22 \\
\hline & $\begin{array}{l}\text { Encuentra relación entre los datos } \\
\text { y las incógnitas. }\end{array}$ & 0 & 22 \\
\hline & $\begin{array}{l}\text { Analiza el problema desde varios } \\
\text { ángulos. }\end{array}$ & 2 & 10 \\
\hline \multicolumn{2}{|c|}{ PROMEDIO } & 2.5 & 18,7 \\
\hline \multicolumn{2}{|c|}{ DESVIACIÓN ESTÁNDAR } & 2,2 & 4,5 \\
\hline \multirow{6}{*}{$\begin{array}{l}\text { Concha Medina de } \\
\text { Silva } \\
9 \text { estudiantes }\end{array}$} & Lee detenidamente la situación. & 1 & 9 \\
\hline & $\begin{array}{l}\text { Determina si conoce el significado } \\
\text { de todas las palabras. }\end{array}$ & & 8 \\
\hline & $\begin{array}{l}\text { Determina qué datos dan y qué } \\
\text { piden. }\end{array}$ & 0 & 9 \\
\hline & Dibuja y/o representa la situación. & 0 & 7 \\
\hline & $\begin{array}{l}\text { Encuentra relación entre los datos } \\
\text { y las incógnitas. }\end{array}$ & 1 & 8 \\
\hline & $\begin{array}{l}\text { Analiza el problema desde varios } \\
\text { ángulos. }\end{array}$ & 1 & 9 \\
\hline \multicolumn{2}{|c|}{ PROMEDIO } & 0,83 & 8,3 \\
\hline \multicolumn{2}{|c|}{ DESVIACIÓN ESTÁNDAR } & 0,7 & 0,8 \\
\hline
\end{tabular}

Fuente: elaboración propia.

Los resultados de los estudiantes que adelantan estudios en las Instituciones Educativas Gustavo Rojas Pinilla(GRP) y Concha Medina de Silva(CMS), sobre resolución de problemas de aplicación matemática, en la etapa de comprensión (ver tabla 2), muestran en el primer caso que en el pretest, la utilización de las subcategorías para el desarrollo del problema fue de $11.4 \% \quad(15 / 132)$, es decir, 15 respuestas de 132 posibles, se observa que en dos subcategorías del pretest no hubo respuesta de los estudiantes, obteniendo una participación media de 2.5; y en el postest la utilización de las subcategorías para el desarrollo del problema fue de 84,8 \% (112/132), observando una notoria evolución en la utilización de estas subcategorías como 
proceso adecuado para la resolución de problemas matemáticos, con una participación media de 18,7 (112/6), con una avance significativo de 73.4 $\%$ y con unas diferencias entre las medias de 16.2. Realizando el mismo análisis en la IE CMS, en el pretest, la utilización de las subcategorías para el desarrollo del problema fue de $9.3 \%$ $(5 / 54)$ que corresponde a 5 respuestas de 54 posibles, observando que en dos subcategorías no hubo respuesta de los estudiantes, con una participación media de 0.83 ; y en el postest fue de $92.6 \%$ (50/54); igualmente, se observa una evolución en la utilización de estas subcategorías en la etapa de comprensión como método adecuado para la resolución de problemas matemáticos, con una participación media de 8.3 (50/6), con un avance significativo del $83.3 \%$ y con una diferencia entre medias de 8.3.

Tabla 3

Etapa de configuración del plan y utilización de subcategorías

\begin{tabular}{|c|c|c|c|}
\hline \multicolumn{4}{|c|}{ Resultados de los estudiantes en la etapa de configurar el plan } \\
\hline \multicolumn{4}{|c|}{ Pre-test } \\
\hline & & & Pos-test \\
\hline $\begin{array}{l}\text { Institución } \\
\text { educativa }\end{array}$ & Subcategoría & Estudiantes & Estudiantes \\
\hline \multirow{6}{*}{$\begin{array}{l}\text { Gustavo Rojas } \\
\text { Pinilla } \\
22 \text { estudiantes }\end{array}$} & Puede utilizar un método empleado con anterioridad. & 1 & 22 \\
\hline & Agrega nuevos elementos para utilizar un método. & 0 & 15 \\
\hline & Son necesarios todos los datos. & 5 & 17 \\
\hline & Usa diferentes estrategias. & 2 & 15 \\
\hline & Revisa su lista de estrategias. & 4 & 22 \\
\hline & Recuerda si ha visto una situación parecida. & 3 & 10 \\
\hline \multicolumn{2}{|l|}{ PROMEDIO } & 2,5 & 16,8 \\
\hline \multicolumn{2}{|c|}{ DESVIACIÓN ESTÁNDAR } & 1,8 & 4,6 \\
\hline \multirow{6}{*}{$\begin{array}{l}\text { Concha Medina } \\
\text { de Silva } \\
9 \text { estudiantes }\end{array}$} & Puede utilizar un método empleado con anterioridad. & 1 & 9 \\
\hline & Agrega nuevos elementos para utilizar un método. & 2 & 7 \\
\hline & Agrega nuevos elementos para utilizar un método. & 1 & 6 \\
\hline & Son necesarios todos los datos. & 1 & 9 \\
\hline & Usa diferentes estrategias. & 1 & 8 \\
\hline & Recuerda si ha visto una situación parecida. & 1 & 9 \\
\hline \multicolumn{2}{|c|}{ PROMEDIO } & 1,2 & 8 \\
\hline \multicolumn{2}{|c|}{ DESVIACIÓN ESTÁNDAR } & 0,4 & 1,2 \\
\hline
\end{tabular}

Fuente: elaboración propia. 
Respecto a la configuración del plan (ver tabla 3), en el pretest, la relación entre los resultados obtenidos de los estudiantes de las dos IE fue de $11.4 / 13.0$ y en el postest de 76.5 / 88.9 , destacando que los que integran la IE CMS, participaron así fuese unitariamente en cada subcategoría, a pesar de las dificultades de comprensión que presentan. En la IE GRP, solo el $45.5 \%$ (10 de los 22) de los estudiantes recuerda haber visto una situación parecida.

Tabla 4

Etapa de aplicación del plan y utilización de subcategorías

\begin{tabular}{|c|c|c|c|}
\hline \multicolumn{4}{|c|}{ Resultados de los estudiantes en la etapa de aplicación del plan } \\
\hline & & \multirow[t]{2}{*}{ Pretest } & \multirow[b]{2}{*}{ Postest } \\
\hline & & & \\
\hline Institución educativa & Subcategoría & Estudiantes & Estudiantes \\
\hline \multirow{5}{*}{$\begin{array}{l}\text { Gustavo Rojas Pinilla } \\
22 \text { estudiantes }\end{array}$} & Establece plan de solución. & 3 & 22 \\
\hline & Comprueba los pasos ya realizados. & 1 & 17 \\
\hline & $\begin{array}{c}\text { Explica lo que hace y para que lo } \\
\text { hace. }\end{array}$ & 2 & 16 \\
\hline & $\begin{array}{c}\text { Soluciona el problema } \\
\text { completamente. }\end{array}$ & 4 & 22 \\
\hline & Reordena las ideas y prueba de nuevo. & 0 & 18 \\
\hline \multicolumn{2}{|c|}{ PROMEDIO } & 2 & 19 \\
\hline \multicolumn{2}{|c|}{ DESVIACIÓN ESTÁNDAR } & 1,5 & 2,8 \\
\hline \multirow{5}{*}{$\begin{array}{c}\text { Concha Medina de Silva } \\
9 \text { estudiantes }\end{array}$} & Establece plan de solución. & 1 & 8 \\
\hline & Comprueba los pasos ya realizados. & 0 & 7 \\
\hline & $\begin{array}{l}\text { Explica lo que hace y para que lo } \\
\text { hace. }\end{array}$ & 0 & 8 \\
\hline & $\begin{array}{c}\text { Soluciona el problema } \\
\text { completamente. }\end{array}$ & 0 & 7 \\
\hline & Reordena las ideas y prueba de nuevo. & 0 & 6 \\
\hline \multicolumn{2}{|c|}{ PROMEDIO } & 0,2 & 7,2 \\
\hline \multicolumn{2}{|c|}{ DESVIACIÓN ESTÁNDAR } & 0,4 & 0,8 \\
\hline
\end{tabular}

Fuente: elaboración propia.

En lo que concierne a los resultados de los estudiantes en la etapa de aplicación del plan, (ver tabla 4), en el pretest los estudiantes de la IE GRP un promedio de 2: $(10 / 5)$ que corresponde a 10 subcategorías usadas correctamente por todos los estudiantes de 5 categorías. En la II CMS, solo usaron una subcategoría, obteniendo así un promedio de 0.2 , mientras que en el postest es de 19 y 7 , respectivamente, mostrando un avance significativo en las dos instituciones. 
Tabla 5

Etapa de revisión, verificación y utilización de subcategorías

Resultados de los estudiantes en la etapa de revisión y verificación

\begin{tabular}{|c|c|c|c|}
\hline \multicolumn{4}{|c|}{ Resultados de los estudiantes en la etapa de revision y verificacion } \\
\hline & & Pretest & Postest \\
\hline $\begin{array}{l}\text { Institución } \\
\text { educativa }\end{array}$ & Subcategoría. & Estudiantes & Estudiantes \\
\hline \multirow{6}{*}{$\begin{array}{l}\text { Gustavo Rojas } \\
\text { Pinilla } \\
22 \text { estudiantes }\end{array}$} & Son correctos los pasos y operaciones. & 2 & 20 \\
\hline & $\begin{array}{l}\text { Establece con precisión cuál fue el paso } \\
\text { clave en la solución. }\end{array}$ & 3 & 19 \\
\hline & $\begin{array}{l}\text { Colabora a sus compañeros para desarrollen } \\
\text { habilidades en la solución de situaciones. }\end{array}$ & 5 & 15 \\
\hline & $\begin{array}{l}\text { Utiliza el resultado obtenido para formular y } \\
\text { plantear nuevas situaciones. }\end{array}$ & 0 & 5 \\
\hline & Se autoevalúa. & 2 & 22 \\
\hline & Lee nuevamente el enunciado y comprueba. & 2 & 22 \\
\hline \multicolumn{2}{|l|}{ PROMEDIO } & 2,3 & 17,3 \\
\hline \multicolumn{2}{|c|}{ DESVIACIÓN ESTÁNDAR } & 1,6 & 6,4 \\
\hline \multirow{6}{*}{$\begin{array}{l}\text { Concha Medina } \\
\text { de Silva } 9 \\
\text { estudiantes }\end{array}$} & Son correctos los pasos y operaciones. & 1 & 9 \\
\hline & $\begin{array}{l}\text { Establece con precisión cuál fue el paso } \\
\text { clave en la solución. }\end{array}$ & 1 & 9 \\
\hline & $\begin{array}{l}\text { Colabora a sus compañeros para desarrollen } \\
\text { habilidades en la solución de situaciones. }\end{array}$ & 1 & 7 \\
\hline & $\begin{array}{l}\text { Utiliza el resultado obtenido para formular y } \\
\text { plantear nuevas situaciones. }\end{array}$ & 0 & 6 \\
\hline & Se autoevalúa. & 1 & 9 \\
\hline & Lee nuevamente el enunciado y comprueba. & 1 & 7 \\
\hline \multicolumn{2}{|l|}{ PROMEDIO } & 0,8 & 7,8 \\
\hline \multicolumn{2}{|c|}{ DESVIACIÓN ESTÁNDAR } & 0,4 & 1,3 \\
\hline
\end{tabular}

Fuente: elaboración propia.

De la misma manera, en la etapa de revisión y verificación, los resultados porcentuales obtenidos respectivamente en las dos IE (ver tabla 5), son los siguientes: el comparativo del promedio pretest-postest en la IE GRP es de 1.6 contra 6.4 ; y en la IE CMS, es de 0.8 contra 7.8 , evidenciando un avance significativo en las dos instituciones.

Situación 2: ¿Cuántos balones en total debe comprar el abuelo para sus nietos, si a cada uno de sus 5 nietos le va a regalar 7 balones? 
Tabla 6

Etapa de comprensión y utilización de subcategorías

\begin{tabular}{|c|c|c|c|}
\hline \multicolumn{4}{|c|}{ Resultados de los estudiantes en la etapa de comprensión } \\
\hline & & Pretest & Postest \\
\hline $\begin{array}{l}\text { Institución } \\
\text { educativa }\end{array}$ & Subcategoría & Estudiantes & Estudiantes \\
\hline \multirow{6}{*}{$\begin{array}{l}\text { Gustavo Rojas } \\
\text { Pinilla } \\
22 \text { estudiantes }\end{array}$} & Lee detenidamente la situación. & 5 & 22 \\
\hline & $\begin{array}{l}\text { Determina si conoce el significado de todas las } \\
\text { palabras. }\end{array}$ & 0 & 22 \\
\hline & Determina qué datos dan y qué piden. & 5 & 22 \\
\hline & Dibuja y/o representa la situación. & 2 & 22 \\
\hline & $\begin{array}{l}\text { Encuentra relación entre los datos y las } \\
\text { incógnitas. }\end{array}$ & 1 & 18 \\
\hline & Analiza el problema desde varios ángulos. & 2 & 12 \\
\hline \multicolumn{2}{|l|}{ PROMEDIO } & 2 & 19,3 \\
\hline \multicolumn{2}{|c|}{ DESVIACIÓN ESTÁNDAR } & 2,0 & 4,0 \\
\hline \multirow{6}{*}{$\begin{array}{l}\text { Concha Medina de } \\
\text { Silva } \\
9 \text { estudiantes }\end{array}$} & Lee detenidamente la situación. & 1 & 9 \\
\hline & $\begin{array}{l}\text { Determina si conoce el significado de todas las } \\
\text { palabras. }\end{array}$ & 0 & 8 \\
\hline & Determina qué datos dan y qué piden. & 2 & 9 \\
\hline & Dibuja y/o representa la situación, & 0 & 9 \\
\hline & $\begin{array}{l}\text { Encuentra relación entre los datos y las } \\
\text { incógnitas. }\end{array}$ & 2 & 7 \\
\hline & Analiza el problema desde varios ángulos. & 3 & 6 \\
\hline \multicolumn{2}{|l|}{ PROMEDIO } & 1,3 & 8 \\
\hline \multicolumn{2}{|c|}{ DESVIACIÓN ESTÁNDAR } & 1,2 & 1,2 \\
\hline
\end{tabular}

Fuente: elaboración propia.

Los resultados de los estudiantes que adelantan estudios en las Instituciones Educativas Gustavo Rojas Pinilla (GRP) y Concha Medina de Silva, sobre resolución de problemas de aplicación matemática, en la etapa de comprensión (ver tabla 6), muestran en el primer caso que en el pretest, la utilización de las subcategorías para el desarrollo del problema fue de 11.4 $\%$ (15/132); es decir, 15 respuestas de 132 posibles, se observa que en una de las subcategorías no hubo respuesta de los estudiantes, obteniendo una participación media de 2 estudiantes; y en el postest de $89.4 \%(118 / 132)$, observando una notoria evolución en la utilización de estas subcategorías como proceso adecuado para la resolución de problemas matemáticos, con una participación media de 20 $(90,9 \%)$, con una avance significativo de 18. Realizando el mismo análisis en la IE CMS, en el pretest, la utilización de las subcategorías para el desarrollo del problema fue de $14.8 \%(8 / 54)$, que correspondea 8 respuestas de 54 posibles, observando que en dos subcategorías no hubo respuesta de los estudiantes, con una participación media de 1.3, y en el postest de $88.9 \%$ (48.54); igualmente, se observa una evolución 
en la utilización de estas subcategorías en la etapa de comprensión como método adecuado para la resolución de problemas matemáticos, con una participación media de 8.0, con una avance significativo de 6.7 , y con una diferencia entre medias de 1.3 .

Tabla 7

Etapa de configuración del plan y utilización de subcategorías

\begin{tabular}{|c|c|c|c|}
\hline \multicolumn{4}{|c|}{ Resultados de los estudiantes en la etapa de configurar el plan } \\
\hline & & Pretest & Postest \\
\hline $\begin{array}{l}\text { Institución } \\
\text { educativa }\end{array}$ & Subcategoría & Estudiantes & Estudiantes \\
\hline \multirow{6}{*}{$\begin{array}{l}\text { Gustavo Rojas } \\
\text { Pinilla } \\
22 \text { estudiantes }\end{array}$} & Puede utilizar un método empleado con anterioridad. & 0 & 20 \\
\hline & Agrega nuevos elementos para utilizar un método. & 0 & 18 \\
\hline & Son necesarios todos los datos. & 5 & 18 \\
\hline & Usa diferentes estrategias. & 3 & 10 \\
\hline & Revisa su lista de estrategias. & 0 & 15 \\
\hline & Recuerda si ha visto una situación parecida. & 0 & 15 \\
\hline \multicolumn{2}{|c|}{ PROMEDIO } & 1,3 & 16 \\
\hline \multicolumn{2}{|c|}{ DESVIACIÓN ESTÁNDAR } & 2,1 & 3,5 \\
\hline \multirow{6}{*}{$\begin{array}{l}\text { Concha Medina de } \\
\text { Silva } \\
9 \text { estudiantes }\end{array}$} & Puede utilizar un método empleado con anterioridad. & 2 & 8 \\
\hline & Agrega nuevos elementos para utilizar un método. & 1 & 7 \\
\hline & Son necesarios todos los datos. & 2 & 9 \\
\hline & Usa diferentes estrategias. & 2 & 8 \\
\hline & Revisa su lista de estrategias. & 2 & 6 \\
\hline & Recuerda si ha visto una situación parecida. & 4 & 8 \\
\hline \multicolumn{2}{|c|}{ PROMEDIO } & 2,1 & 7,6 \\
\hline \multicolumn{2}{|c|}{ DESVIACIÓN ESTÁNDAR } & 1 & 1 \\
\hline
\end{tabular}

Fuente: elaboración propia.

Respecto a la configuración del plan, en el pretest, la relación entre los resultados obtenidos de los estudiantes de las dos IE (ver tabla 7), fue de 6,1 /24.1; y en el postest, de 72.7 / 85.2, destacando que los que integran la IE CMS, tuvieron mayor participación en todas las subcategorías, a pesar de las dificultades de comprensión que presentan, obteniendo a su favor una diferencia porcentual frente a los de la GRP, de 12.5. En la IE GRP, solo el $45.5 \%$ (10) de los estudiantes no usa diferentes estrategias, ante un $54.5 \%$ (12) que sí lo hace. 
Tabla 8

Etapa de aplicación del plan y utilización de subcategorías

\begin{tabular}{|c|c|c|c|}
\hline \multicolumn{4}{|c|}{ Resultados de los estudiantes en la etapa de aplicación del plan } \\
\hline & & \multirow{2}{*}{\multicolumn{2}{|c|}{$\begin{array}{l}\text { Pretest } \\
\\
\text { Postest }\end{array}$}} \\
\hline & & & \\
\hline Institución educativa & Subcategoría & Estudiantes & Estudiantes \\
\hline \multirow{5}{*}{$\begin{array}{l}\text { Gustavo Rojas Pinilla } \\
22 \text { estudiantes }\end{array}$} & Establece plan de solución. & 0 & 22 \\
\hline & Comprueba los pasos ya realizados. & 0 & 18 \\
\hline & Explica lo que hace y para que lo hace. & 2 & 16 \\
\hline & Soluciona el problema completamente. & 5 & 22 \\
\hline & Reordena las ideas y prueba de nuevo. & 0 & 10 \\
\hline \multicolumn{2}{|c|}{ PROMEDIO } & 1,4 & 17,6 \\
\hline \multicolumn{2}{|c|}{ DESVIACIÓN ESTÁNDAR } & 2 & 5 \\
\hline \multirow{5}{*}{$\begin{array}{l}\text { Concha Medina de } \\
\text { Silva } \\
9 \text { estudiantes }\end{array}$} & Establece plan de solución. & 2 & 9 \\
\hline & Comprueba los pasos ya realizados. & 2 & 6 \\
\hline & Explica lo que hace y para que lo hace. & 1 & 5 \\
\hline & Soluciona el problema completamente & 1 & 9 \\
\hline & Reordena las ideas y prueba de nuevo. & 0 & 7 \\
\hline \multicolumn{2}{|c|}{ PROMEDIO } & 1,2 & 7,2 \\
\hline \multicolumn{2}{|c|}{ DESVIACIÓN ESTÁNDAR } & 0,8 & 1,7 \\
\hline
\end{tabular}

Fuente: elaboración propia.

En lo que concierne a los resultados de los estudiantes en la etapa de aplicación del plan, la relación comparativa de los resultados de las IE (ver tabla 8), es el siguiente: el promedio de subcategorías utilizadas por los estudiantes en el pretest fue de 1.4, mientras que en el postest fue 17.6. Se destaca que $10(45.5$ \%) de los estudiantes de la IE GRP, no reordenan las ideas y por tanto no prueban de nuevo.

\section{Tabla 9}

\section{Etapa de revisión, verificación y utilización de subcategorías}

\begin{tabular}{|c|c|c|c|}
\hline & \multicolumn{3}{|c|}{ Resultados de los estudiantes en la etapa de revisión y verificación } \\
\hline & & Pretest & Postest \\
\hline $\begin{array}{l}\text { Institución } \\
\text { educativa }\end{array}$ & Subcategoría & Estudiantes & Estudiantes \\
\hline \multirow{6}{*}{$\begin{array}{l}\text { Gustavo Rojas } \\
\text { Pinilla } \\
22 \text { estudiantes }\end{array}$} & Son correctos los pasos y operaciones. & 5 & 20 \\
\hline & $\begin{array}{l}\text { Establece con precisión cuál fue el paso clave en la } \\
\text { solución. }\end{array}$ & 0 & 19 \\
\hline & $\begin{array}{l}\text { Colabora a sus compañeros para desarrollen habilidades } \\
\text { en la solución de situaciones. }\end{array}$ & 0 & 15 \\
\hline & $\begin{array}{l}\text { Utiliza el resultado obtenido para formular y plantear } \\
\text { nuevas situaciones. }\end{array}$ & 0 & 10 \\
\hline & Se autoevalúa. & 2 & 22 \\
\hline & Lee nuevamente el enunciado y comprueba. & 2 & 22 \\
\hline
\end{tabular}




\begin{tabular}{llcc}
\hline PROMEDIO & \multicolumn{1}{c}{1,5} & 18 \\
\hline DESVIACIÓN ESTÁNDAR & 2 & 4,6 \\
\hline $\begin{array}{l}\text { Concha Medina de } \\
\text { Silva 9 estudiantes }\end{array}$ & Son correctos los pasos y operaciones. & 1 & 9 \\
\cline { 2 - 4 } & $\begin{array}{l}\text { Establece con precisión cuál fue el paso clave en la } \\
\text { solución. }\end{array}$ & 2 & 9 \\
\cline { 2 - 4 } & $\begin{array}{l}\text { Colabora a sus compañeros para desarrollen habilidades } \\
\text { en la solución de situaciones. }\end{array}$ & 2 & 5 \\
\cline { 2 - 4 } & $\begin{array}{l}\text { Utiliza el resultado obtenido para formular y plantear } \\
\text { nuevas situaciones. }\end{array}$ & 2 & 5 \\
\cline { 2 - 4 } & Se autoevalúa. & 2 & 7 \\
\cline { 2 - 4 } & Lee nuevamente el enunciado y comprueba. & 1 & 8 \\
\hline PROMEDIO & & 1,6 & 7,1 \\
\hline DESVIACIÓN ESTÁNDAR & 0,5 & 1,8 \\
\hline
\end{tabular}

Fuente: elaboración propia.

De la misma manera, en la etapa de revisión y verificación (ver tabla 9), los resultados porcentuales obtenidos respectivamente en las dos IE, son los siguientes: 7,6 / 18,5 y 81,8 / 79,6, observándose una diferencia porcentual positiva de 4 puntos a favor de la IE GRP, porque la relación de participación estudiantil es de 18 $(81.8 \%)$ y de 7 (77,8 \%), lo cual se justifica en la notoria disminución en dos subcategorías: colabora a sus compañeros para que desarrollen habilidades en la solución de situaciones (5 estudiantes) y utiliza el resultado obtenido para formular y plantear nuevas situaciones (5 estudiantes).

Situación 3: Un día tiene 24 horas ¿cuántas horas tiene cinco días?

\section{Tabla 10}

Etapa de comprensión y utilización de subcategorías

\begin{tabular}{|c|c|c|c|}
\hline \multicolumn{4}{|c|}{ Resultados de los estudiantes en la etapa de comprensión } \\
\hline & & Pretest & Postest \\
\hline $\begin{array}{l}\text { Institución } \\
\text { educativa }\end{array}$ & Subcategoría & Estudiantes & Estudiantes \\
\hline \multirow{6}{*}{$\begin{array}{l}\text { Gustavo Rojas } \\
\text { Pinilla } \\
22 \text { estudiantes }\end{array}$} & Lee detenidamente la situación. & 4 & 22 \\
\hline & Determina si conoce el significado de todas las palabras. & 0 & 20 \\
\hline & Determina qué datos dan y qué piden. & 5 & 20 \\
\hline & Dibuja y/o representa la situación. & 3 & 22 \\
\hline & Encuentra relación entre los datos y las incógnitas. & 0 & 22 \\
\hline & Analiza el problema desde varios ángulos. & 2 & 10 \\
\hline PROMEDIO & & 2 & 19 \\
\hline DESVIACIÓN E & TÁNDAR & 2 & 4,6 \\
\hline \multirow{6}{*}{$\begin{array}{l}\text { Concha Medina } \\
\text { de Silva } \\
9 \text { estudiantes }\end{array}$} & Lee detenidamente la situación. & 1 & 9 \\
\hline & Determina si conoce el significado de todas las palabras. & 2 & 9 \\
\hline & Determina qué datos dan y qué piden. & 2 & 7 \\
\hline & Dibuja y/o representa la situación. & 0 & 7 \\
\hline & Encuentra relación entre los datos y las incógnitas. & 2 & 9 \\
\hline & Analiza el problema desde varios ángulos. & 1 & 6 \\
\hline \multicolumn{2}{|l|}{ PROMEDIO } & 1,3 & 7,8 \\
\hline \multicolumn{2}{|c|}{ DESVIACIÓN ESTÁNDAR } & 1 & 1,3 \\
\hline
\end{tabular}

Fuente: elaboración propia. 
Los resultados de los estudiantes que adelantan estudios en las Instituciones Educativas Gustavo Rojas Pinilla (GRP) y Concha Medina de Silva (CMS), sobre resolución de problemas de aplicación matemática, en la etapa de comprensión (ver tabla 10), muestran en el primer caso que en el pretest, la utilización de las subcategorías para el desarrollo del problema fue de 10.6 $\%$ (15/132), es decir, 15 respuestas de 132 posibles; se observa que en dos subcategorías del pretest no hubo respuesta de los estudiantes, obteniendo una participación media de 2 estudiantes (9.1); y en el postest, la utilización de las subcategorías para el desarrollo del problema fue de 87.9 $\%$ (116/132), observando una notoria evolución en la utilización de estas subcategorías como proceso adecuado para la resolución de problemas matemáticos, con una participación media de 19 (86.4\%), con una avance significativo de $77.3 \%$.

Realizando el mismo análisis en la IE CMS, en el pretest, la utilización de las subcategorías para el desarrollo del problema fue de $14.8 \%(8 / 54)$, que corresponde a 8 respuestas de 54 posibles, observando que en una subcategoría no hubo respuesta de los estudiantes, con una participación media de 1.1 (8/54); y en el postest, fue de $87.0 \%(47 / 54)$; igualmente, se observa una evolución en la utilización de estas subcategorías en la etapa de comprensión como método adecuado para la resolución de problemas matemáticos, con una participación media de 8.3 (50/6), con un avance significativo del $83.3 \%$ y con una diferencia entre medias de 8.8.

\section{Tabla 11}

Etapa de configuración del plan y utilización de subcategorías

\begin{tabular}{|c|c|c|c|}
\hline \multicolumn{4}{|c|}{ Resultados de los estudiantes en la etapa de configurar el plan } \\
\hline & & Pretest & Postest \\
\hline Institución educativa & Subcategoría & Estudiantes & Estudiantes \\
\hline \multirow{6}{*}{$\begin{array}{l}\text { Gustavo Rojas Pinilla } \\
22 \text { estudiantes }\end{array}$} & Puede utilizar un método empleado con anterioridad. & 1 & 22 \\
\hline & Agrega nuevos elementos para utilizar un método. & 0 & 15 \\
\hline & Son necesarios todos los datos. & 5 & 22 \\
\hline & Usa diferentes estrategias. & 2 & 15 \\
\hline & Revisa su lista de estrategias. & 0 & 22 \\
\hline & Recuerda si ha visto una situación parecida. & 2 & 15 \\
\hline \multicolumn{2}{|c|}{ PROMEDIO } & 1,6 & 18,5 \\
\hline \multicolumn{2}{|c|}{ DESVIACIÓN ESTÁNDAR } & 2 & 4 \\
\hline \multirow{6}{*}{$\begin{array}{l}\text { Concha Medina de } \\
\text { Silva } \\
9 \text { estudiantes }\end{array}$} & Puede utilizar un método empleado con anterioridad. & 2 & 9 \\
\hline & Agrega nuevos elementos para utilizar un método. & 0 & 6 \\
\hline & Son necesarios todos los datos. & 1 & 9 \\
\hline & Usa diferentes estrategias. & 1 & 6 \\
\hline & Revisa su lista de estrategias. & 2 & 7 \\
\hline & Recuerda si ha visto una situación parecida. & 2 & 9 \\
\hline \multicolumn{2}{|l|}{ PROMEDIO } & 1,3 & 7,6 \\
\hline \multicolumn{2}{|c|}{ DESVIACIÓN ESTÁNDAR } & 1 & 1,5 \\
\hline
\end{tabular}

Fuente: elaboración propia. 
Respecto a la configuración del plan, en el pretest, la relación entre los resultados obtenidos de los estudiantes de las dos IE (ver tabla 11), fue de $10.6 / 14,8$; y en el postest, de 84.1 / 85.2, destacando que los que integran la IE CMS, tuvieron mayor participación en todas las subcategorías, a pesar de las dificultades de comprensión que presentan, obteniendo a su favor una diferencia porcentual frente a los de la GRP, de 1.1. En la IE GRP, solo los 15 estudiantes $(68,2 \%)$ no agregan nuevos elementos para utilizar un método, utiliza las mismas estrategias de siempre y no recuerda haber visto una situación parecida.

Tabla 12

Etapa de aplicación del plan y utilización de subcategorías

\begin{tabular}{llcc}
\hline \multicolumn{4}{c}{ Resultados de los estudiantes en la etapa de aplicación del plan } \\
\hline \multirow{2}{*}{ Institución educativa } & Subcategoría & Pretest & Postest \\
\hline \multirow{2}{*}{$\begin{array}{l}\text { Gustavo Rojas Pinilla } \\
\text { estudiantes }\end{array}$} & Establece plan de solución. & 5 & 22 \\
\cline { 2 - 4 } & Comprueba los pasos ya realizados. & 0 & 18 \\
\cline { 2 - 4 } & Explica lo que hace y para qué lo hace. & 2 & 18 \\
\cline { 2 - 4 } & Soluciona el problema completamente. & 4 & 22 \\
\cline { 2 - 4 } & Reordena las ideas y prueba de nuevo. & 0 & 18 \\
\hline PROMEDIO & & 2,2 & 19,6 \\
\hline DESVIACIÓN ESTÁNDAR & 1,2 & 2 \\
\hline Concha Medina de & Establece plan de solución. & 2 & 9 \\
\cline { 2 - 4 } 9 estudiantes & Comprueba los pasos ya realizados. & 1 & 7 \\
\cline { 2 - 4 } & Explica lo que hace y para que lo hace. & 3 & 6 \\
\cline { 2 - 4 } & Soluciona el problema completamente. & 2 & 7 \\
\cline { 2 - 4 } & Reordena las ideas y prueba de nuevo. & 1 & 1,3 \\
\hline PROMEDIO & & 1 & 9 \\
\hline DESVIACIÓN ESTÁNDAR & & 1,8 \\
\hline
\end{tabular}

Fuente: elaboración propia.

En lo que concierne a los resultados de los estudiantes en la etapa de aplicación del plan, (ver tabla 12), la relación comparativa de los resultados de las IE, es el siguiente: 10,0/20,0 en el pretest; mientras que, en el postest, es de 89.1 / 84.4, con una diferencia negativa para los estudiantes de la IE CMS de -4.7, en la primera fase porque los de la IE GRP, tuvieron mayor participación.

Tabla 13

Etapa de revisión, verificación y utilización de subcategorías

\begin{tabular}{|c|c|c|c|}
\hline & \multicolumn{3}{|c|}{ Resultados de los estudiantes en la etapa de revisión y verificación } \\
\hline & & Pretest & Postest \\
\hline Institución educativa & Subcategoría & Estudiantes & Estudiantes \\
\hline \multirow{6}{*}{$\begin{array}{l}\text { Gustavo Rojas Pinilla } \\
22 \text { estudiantes }\end{array}$} & Son correctos los pasos y operaciones. & 3 & 22 \\
\hline & Establece con precisión cuál fue el paso clave en la solución. & 3 & 19 \\
\hline & $\begin{array}{l}\text { Colabora a sus compañeros para desarrollen habilidades en la } \\
\text { solución de situaciones. }\end{array}$ & 0 & 15 \\
\hline & $\begin{array}{l}\text { Utiliza el resultado obtenido para formular y plantear nuevas } \\
\text { situaciones, }\end{array}$ & 0 & 5 \\
\hline & Se autoevalúa. & 2 & 22 \\
\hline & Lee nuevamente el enunciado y comprueba. & 2 & 22 \\
\hline
\end{tabular}




\begin{tabular}{|c|c|c|c|}
\hline \multicolumn{2}{|c|}{ PROMEDIO } & 1,6 & 18 \\
\hline \multicolumn{2}{|c|}{ DESVIACIÓN ESTÁNDAR } & 1,3 & 6,7 \\
\hline \multirow{6}{*}{$\begin{array}{l}\text { Concha Medina de } \\
\text { Silva } 9 \text { estudiantes }\end{array}$} & Son correctos los pasos y operaciones. & 1 & 9 \\
\hline & Establece con precisión cuál fue el paso clave en la solución- & 1 & 7 \\
\hline & $\begin{array}{l}\text { Colabora a sus compañeros para desarrollen habilidades en la } \\
\text { solución de situaciones. }\end{array}$ & 2 & 6 \\
\hline & $\begin{array}{l}\text { Utiliza el resultado obtenido para formular y plantear nuevas } \\
\text { situaciones. }\end{array}$ & 1 & 5 \\
\hline & Se autoevalúa. & 1 & 6 \\
\hline & Lee nuevamente el enunciado y comprueba. & 0 & 7 \\
\hline \multicolumn{2}{|c|}{ PROMEDIO } & 1,3 & 7,6 \\
\hline \multicolumn{2}{|c|}{ DESVIACIÓN ESTÁNDAR } & 1 & 1,5 \\
\hline
\end{tabular}

Fuente: elaboración propia.

De la misma manera, en la etapa de revisión y verificación, los resultados porcentuales obtenidos respectivamente en las dos IE (ver tabla 13), son los siguientes: 7.6 / 11.1 y 79.5 / 74.1, observándose una diferencia porcentual positiva de 5.4 puntos a favor de la IE GRP, porque la relación de participación estudiantil es de $18(81.8 \%)$ y de $7(77.8$ $\%$ ), lo cual se justifica en la notoria disminución en la subcategoría: utiliza el resultado obtenido para formular y plantear nuevas situaciones (5 estudiantes, $22.7 \%$ ).

Situación 4: Don Pedro tiene una parcela de lechugas de forma cuadrada, si el área de la parcela es de 16 metros cuadrados ¿Cuál es la longitud del lado de la parcela?

Tabla 14

Etapa de comprensión y utilización de subcategorías

\begin{tabular}{|c|c|c|c|}
\hline \multicolumn{4}{|c|}{ Resultados de los estudiantes en la etapa de comprensión } \\
\hline & & Pretest & Postest \\
\hline Institución educativa & Subcategoría & Estudiantes & Estudiantes \\
\hline \multirow{6}{*}{$\begin{array}{l}\text { Gustavo Rojas Pinilla } \\
22 \text { estudiantes }\end{array}$} & Lee detenidamente la situación. & 3 & 15 \\
\hline & Determina si conoce el significado de todas las palabras. & 0 & 18 \\
\hline & Determina qué datos dan y qué piden. & 5 & 22 \\
\hline & Dibuja y/o representa la situación. & 3 & 22 \\
\hline & Encuentra relación entre los datos y las incógnitas. & 0 & 22 \\
\hline & Analiza el problema desde varios ángulos. & 2 & 18 \\
\hline \multicolumn{2}{|l|}{ PROMEDIO } & 2 & 19,5 \\
\hline \multicolumn{2}{|c|}{ DESVIACIÓN ESTÁNDAR } & 2 & 3 \\
\hline \multirow{6}{*}{$\begin{array}{l}\text { Concha Medina de } \\
\text { Silva } \\
9 \text { estudiantes }\end{array}$} & Lee detenidamente la situación. & 1 & 9 \\
\hline & Determina si conoce el significado de todas las palabras. & 0 & 7 \\
\hline & Determina qué datos dan y qué piden. & 0 & 8 \\
\hline & Dibuja y/o representa la situación. & 2 & 9 \\
\hline & Encuentra relación entre los datos y las incógnitas. & 0 & 7 \\
\hline & Analiza el problema desde varios ángulos. & 2 & 8 \\
\hline \multicolumn{2}{|l|}{ PROMEDIO } & 1 & 8 \\
\hline \multicolumn{2}{|c|}{ DESVIACIÓN ESTÁNDAR } & 1 & 1 \\
\hline
\end{tabular}

Fuente: elaboración propia. 
Los resultados de los estudiantes que adelantan estudios en las Instituciones Educativas Gustavo Rojas Pinilla (GRP) y Concha Medina de Silva (CMS), sobre resolución de problemas de aplicación matemática (ver tabla 14), en la etapa de comprensión, muestran en el primer caso que en el pretest, la utilización de las subcategorías para el desarrollo del problema fue de 9,8 $\%(13 / 132)$, es decir, 13 respuestas de 132 posibles; se observa que en dos subcategorías del pretest no hubo respuesta de los estudiantes, obteniendo una participación media de 2.0; y en el postest, la utilización de las subcategorías para el desarrollo del problema fue de 88,6 \% (117/132), observando una notoria evolución en la utilización de estas subcategorías como proceso adecuado para la resolución de problemas matemáticos, con una participación media de 19 $(86.3 \%)$, con una avance significativo de $77.34 \%$ y con unas diferencias entre las medias de 17,0 .

Realizando el mismo análisis en la IE CMS, en el pretest, la utilización de las subcategorías para el desarrollo del problema fue de $9.3 \%(5 / 54)$, que corresponde a 5 respuestas de 54 posibles, observando que en tres subcategorías no hubo respuesta de los estudiantes, con una participación media de 1.0; y en el postest fue de $88.9 \%$ (48/54); igualmente, se observa una evolución en la utilización de estas subcategorías en la etapa de comprensión como método adecuado para la resolución de problemas matemáticos, con una participación media de $8.3(50 / 6)$, con un avance significativo del $83.3 \%$ y con una diferencia entre medias de 8.3.

Tabla 15

Etapa de configuración del plan y utilización de subcategorías

\begin{tabular}{|c|c|c|c|}
\hline \multicolumn{4}{|c|}{ Resultados de los estudiantes en la etapa de configurar el plan } \\
\hline & & Pretest & Postest \\
\hline Institución educativa & Subcategoría & Estudiantes & Estudiantes \\
\hline \multirow{6}{*}{$\begin{array}{l}\text { Gustavo Rojas Pinilla } \\
22 \text { estudiantes }\end{array}$} & Puede utilizar un método empleado con anterioridad. & 1 & 22 \\
\hline & Agrega nuevos elementos para utilizar un método. & 0 & 15 \\
\hline & Son necesarios todos los datos. & 0 & 17 \\
\hline & Usa diferentes estrategias. & 2 & 18 \\
\hline & Revisa su lista de estrategias. & 0 & 22 \\
\hline & Recuerda si ha visto una situación parecida. & 3 & 10 \\
\hline \multicolumn{2}{|l|}{ PROMEDIO } & 1 & 17 \\
\hline \multicolumn{2}{|c|}{ DESVIACIÓN ESTÁNDAR } & 1 & 5 \\
\hline \multirow{6}{*}{$\begin{array}{l}\text { Concha Medina de Silva } \\
9 \text { estudiantes }\end{array}$} & Puede utilizar un método empleado con anterioridad. & 1 & 6 \\
\hline & Agrega nuevos elementos para utilizar un método. & 0 & 5 \\
\hline & Son necesarios todos los datos. & 0 & 7 \\
\hline & Usa diferentes estrategias. & 1 & 7 \\
\hline & Revisa su lista de estrategias. & 1 & 5 \\
\hline & Recuerda si ha visto una situación parecida. & 1 & 9 \\
\hline \multicolumn{2}{|l|}{ PROMEDIO } & 0,6 & 6,5 \\
\hline \multicolumn{2}{|c|}{ DESVIACIÓN ESTÁNDAR } & 1 & 2 \\
\hline
\end{tabular}

Fuente: elaboración propia. 
Respecto a la configuración del plan, en el pretest, la relación entre los resultados obtenidos de los estudiantes de las dos IE (ver tabla 15), fue de 4.5 /9.3; y en el postest, de 78.8 / 72.2, destacando que los que integran la IE GRP, tuvieron mayor participación en todas las subcategorías, a pesar de las dificultades de comprensión que presentan; sin embargo, presentan una diferencia porcentual en contra frente a los de la CMS, de 6.6. En la IE GRP, solo 10 estudiantes (45.5\%) no recuerdan haber visto una situación parecida. A pesar de que ambos grupos de participantes mostraron dificultades al resolver el pre test en varias subcategorías, los mayores problemas los enfrentaron los que hacen parte de la CMS, que no se repusieron en la segunda fase.

\section{Tabla 16}

Etapa de aplicación del plan y utilización de subcategorías

\begin{tabular}{llll}
\hline \multicolumn{4}{c}{ Resultados de los estudiantes en la etapa de aplicación del plan } \\
\hline & & \multicolumn{1}{c}{ Pretest } & Postest \\
\hline Institución educativa & Subcategoría & \multicolumn{1}{c}{ Estudiantes } & Estudiantes \\
\hline Gustavo Rojas Pinilla & Establece plan de solución. & 3 & 22 \\
\cline { 2 - 4 } 22 estudiantes & Comprueba los pasos ya realizados. & 1 & 17 \\
\cline { 2 - 4 } & Explica lo que hace y para qué lo hace. & 2 & 14 \\
\cline { 2 - 4 } & Soluciona el problema completamente. & 4 & 22 \\
\cline { 2 - 4 } & Reordena las ideas y prueba de nuevo. & 0 & 18 \\
\hline PROMEDIO & & 2 & 18,6 \\
\hline DESVIACIÓN ESTÁNDAR & 2 & 3,4 \\
\hline Concha Medina de & Establece plan de solución. & 1 & 9 \\
\cline { 2 - 4 } $\begin{array}{l}\text { Silva } \\
\text { 9 estudiantes }\end{array}$ & Comprueba los pasos ya realizados. & 0 & 4 \\
\cline { 2 - 4 } & Explica lo que hace y para qué lo hace. & 1 & 7 \\
\cline { 2 - 4 } & Soluciona el problema completamente. & 0 & 7,2 \\
\cline { 2 - 4 } & Reordena las ideas y prueba de nuevo. & 0 & 2 \\
\hline PROMEDIO & & 0,4 & 7 \\
\hline DESVIACIÓN ESTÁNDAR & & 1 & 7 \\
\hline
\end{tabular}

Fuente: elaboración propia.

En los resultados de los estudiantes en la etapa de aplicación del plan, la relación comparativa de los resultados de las IE (ver tabla 16), es el siguiente: $9.1 / 4.4$ en el pretest, mientras que en el postest es de $84.5 / 97.8$, con una diferencia negativa para los estudiantes de la IE GRP de -13.3; en la primera fase, estos mostraron mayor participación. Nuevamente, se observan cuatro subcategorías, en las que no hubo participación de los estudiantes de la IE CMS. 
Tabla 17

Etapa de revisión, verificación y utilización de subcategorías

\begin{tabular}{|c|c|c|c|}
\hline \multicolumn{4}{|c|}{ Resultados de los estudiantes en la etapa de revisión y verificación } \\
\hline & & Pretest & Postest \\
\hline Institución educativa & Subcategoría & Estudiantes & Estudiantes \\
\hline \multirow{6}{*}{$\begin{array}{l}\text { Gustavo Rojas Pinilla } \\
22 \text { estudiantes }\end{array}$} & Son correctos los pasos y operaciones. & 2 & 20 \\
\hline & $\begin{array}{l}\text { Establece con precisión cuál fue el paso clave en la } \\
\text { solución. }\end{array}$ & 3 & 15 \\
\hline & $\begin{array}{l}\text { Colabora a sus compañeros para desarrollen habilidades } \\
\text { en la solución de situaciones. }\end{array}$ & 5 & 15 \\
\hline & $\begin{array}{l}\text { Utiliza el resultado obtenido para formular y plantear } \\
\text { nuevas situaciones. }\end{array}$ & 0 & 8 \\
\hline & Se autoevalúa. & 2 & 22 \\
\hline & Lee nuevamente el enunciado y comprueba. & 2 & 22 \\
\hline \multicolumn{2}{|c|}{ PROMEDIO } & 2,3 & 17 \\
\hline \multicolumn{2}{|c|}{ DESVIACIÓN ESTÁNDAR } & 2 & 5 \\
\hline \multirow{6}{*}{$\begin{array}{l}\text { Concha Medina de } \\
\text { Silva } 9 \text { estudiantes }\end{array}$} & Son correctos los pasos y operaciones. & 0 & 9 \\
\hline & $\begin{array}{l}\text { Establece con precisión cuál fue el paso clave en la } \\
\text { solución. }\end{array}$ & 1 & 7 \\
\hline & $\begin{array}{l}\text { Colabora a sus compañeros para desarrollen habilidades } \\
\text { en la solución de situaciones. }\end{array}$ & 1 & 8 \\
\hline & $\begin{array}{l}\text { Utiliza el resultado obtenido para formular y plantear } \\
\text { nuevas situaciones. }\end{array}$ & 0 & 5 \\
\hline & Se autoevalúa. & 1 & 9 \\
\hline & Lee nuevamente el enunciado y comprueba. & 0 & 6 \\
\hline \multicolumn{2}{|c|}{ PROMEDIO } & 0,5 & 7,3 \\
\hline \multicolumn{2}{|c|}{ DESVIACIÓN ESTÁNDAR } & 1 & 2 \\
\hline
\end{tabular}

Fuente: elaboración propia.

De la misma manera, en la etapa de revisión y verificación, los resultados porcentuales obtenidos respectivamente en las dos IE (ver tabla 17), son los siguientes: $10.6 /$ 5.6 y 77.3 / 81.5, observándose una diferencia porcentual positiva de 4,2 puntos a favor de la IE CMS, porque la relación de participación estudiantil es de $17(77.3 \%)$ y de 7 (77.8 \%), lo cual se justifica en la notoria disminución en la subcategoría: utiliza el resultado obtenido para formular y plantear nuevas situaciones (8 estudiantes, 36.4 $0)$.

Situación 5: En una tienda se vendieron 20 helados a $\$ 500$ cada uno ¿Cuál fue la ganancia por cada helado, si los 20 helados le costaron $\$ 8.000$ ? 
Tabla 18

Etapa de comprensión y utilización de subcategorías

\begin{tabular}{|c|c|c|c|}
\hline \multicolumn{4}{|c|}{ Resultados de los estudiantes en la etapa de comprensión } \\
\hline & & Pretest & Postest \\
\hline Institución educativa & Subcategoría & Estudiantes & Estudiantes \\
\hline \multirow{6}{*}{$\begin{array}{l}\text { Gustavo Rojas Pinilla } \\
22 \text { estudiantes }\end{array}$} & Lee detenidamente la situación. & 3 & 22 \\
\hline & Determina si conoce el significado de todas las palabras. & 0 & 18 \\
\hline & Determina qué datos dan y qué piden. & 5 & 18 \\
\hline & Dibuja y/o representa la situación. & 3 & 22 \\
\hline & Encuentra relación entre los datos y las incógnitas. & 0 & 22 \\
\hline & Analiza el problema desde varios ángulos. & 2 & 20 \\
\hline \multicolumn{2}{|c|}{ PROMEDIO } & 2 & 20 \\
\hline \multicolumn{2}{|c|}{ DESVIACIÓN ESTÁNDAR } & 2 & 2 \\
\hline \multirow{6}{*}{$\begin{array}{l}\text { Concha Medina de } \\
\text { Silva } \\
9 \text { estudiantes }\end{array}$} & Lee detenidamente la situación. & 1 & 9 \\
\hline & Determina si conoce el significado de todas las palabras. & 2 & 7 \\
\hline & Determina qué datos dan y qué piden. & 1 & 8 \\
\hline & Dibuja y/o representa la situación. & 2 & 9 \\
\hline & Encuentra relación entre los datos y las incógnitas. & 1 & 7 \\
\hline & Analiza el problema desde varios ángulos. & 2 & 6 \\
\hline \multicolumn{2}{|c|}{ PROMEDIO } & 2 & 8 \\
\hline \multicolumn{2}{|c|}{ DESVIACIÓN ESTÁNDAR } & 1 & 1 \\
\hline
\end{tabular}

Fuente: elaboración propia.

Los resultados de los estudiantes que adelantan estudios en las Instituciones Educativas Gustavo Rojas Pinilla(GRP) y Concha Medina de Silva(CMS), sobre resolución de problemas de aplicación matemática (ver tabla 18), en la etapa de comprensión, muestran en el primer caso que en el pretest, la utilización de las subcategorías para el desarrollo del problema fue de $9.8 \%(13 / 132)$, es decir, 13 respuestas de 132 posibles; se observa que en dos subcategorías del pretest no hubo respuesta de los estudiantes, obteniendo una participación media de 2 estudiantes; $\mathrm{y}$ en el postest, la utilización de las subcategorías para el desarrollo del problema fue de $88.6 \%(122 / 132)$, observando una notoria evolución en la utilización de estas subcategorías como proceso adecuado para la resolución de problemas matemáticos, con una participación media de $20.0(120 / 6)$, con una avance significativo de 90.9 $\%$ y con unas diferencias entre las medias de 18.0. Realizando el mismo análisis en la IE CMS, en el pretest, la utilización de las subcategorías para el desarrollo del problema fue de $14.8 \%(8 / 54)$, que corresponde a 8 respuestas de 54 posibles, observando que en todas las subcategorías hubo respuesta de los estudiantes, con una participación media de 1.0; y en el postest, fue de $88.9 \%$ (48/54); igualmente, se observa una evolución en la utilización de estas subcategorías en la etapa de comprensión como método adecuado para la resolución de problemas matemáticos, con una participación media de $8.3(50 / 6)$, con un avance significativo del $83.3 \%$ y con una diferencia entre medias de 8.3. 
Tabla 19

Etapa de configuración del plan y utilización de subcategorías

\begin{tabular}{|c|c|c|c|}
\hline \multicolumn{4}{|c|}{ Resultados de los estudiantes en la etapa de configurar el plan } \\
\hline & & Pretest & Postest \\
\hline Institución educativa & Categoría & Estudiantes & Estudiantes \\
\hline \multirow{6}{*}{$\begin{array}{l}\text { Gustavo Rojas Pinilla } \\
22 \text { estudiantes }\end{array}$} & Puede utilizar un método empleado con anterioridad. & 1 & 16 \\
\hline & Agrega nuevos elementos para utilizar un método. & 0 & 15 \\
\hline & Son necesarios todos los datos. & 0 & 17 \\
\hline & Usa diferentes estrategias. & 2 & 18 \\
\hline & Revisa su lista de estrategias. & 0 & 22 \\
\hline & Recuerda si ha visto una situación parecida. & 3 & 10 \\
\hline \multicolumn{2}{|c|}{ PROMEDIO } & 1 & 16 \\
\hline \multicolumn{2}{|c|}{ DESVIACIÓN ESTÁNDAR } & 1 & 4 \\
\hline \multirow{6}{*}{$\begin{array}{l}\text { Concha Medina de } \\
\text { Silva } \\
9 \text { estudiantes }\end{array}$} & Puede utilizar un método empleado con anterioridad. & 1 & 6 \\
\hline & Agrega nuevos elementos para utilizar un método. & 1 & 5 \\
\hline & Son necesarios todos los datos. & 1 & 8 \\
\hline & Usa diferentes estrategias. & 2 & 6 \\
\hline & Revisa su lista de estrategias. & 1 & 9 \\
\hline & Recuerda si ha visto una situación parecida. & 1 & 7 \\
\hline \multicolumn{2}{|c|}{ PROMEDIO } & 1 & 7 \\
\hline \multicolumn{2}{|c|}{ DESVIACIÓN ESTÁNDAR } & 0,4 & 1,4 \\
\hline
\end{tabular}

Fuente: elaboración propia.

Respecto a la configuración del plan, en el pretest, la relación entre los resultados obtenidos de los estudiantes de las dos IE (ver tabla 19), fue de 4.5 /13.0; y en el postest, de 74.2 / 75.9, destacando que los que integran la IE CMS, tuvieron mayor participación en todas las subcategorías, a pesar de las dificultades de comprensión que presentan; sin embargo, presentan una diferencia porcentual positiva en contra frente a los de la CRP, de 1.7. En la IE GRP, solo 10 estudiantes $(45.5 \%)$ no recuerdan haber visto una situación parecida. A pesar de que ambos grupos de participantes mostraron dificultades al resolver el pretest en varias subcategorías, los mayores problemas los enfrentaron los que hacen parte de la GRP, que no se repusieron en la segunda fase.

Tabla 20

Etapa de aplicación del plan y utilización de subcategorías

\begin{tabular}{llcc}
\hline & \multicolumn{2}{c}{ Resultados de los estudiantes en la etapa de aplicación del plan } \\
\hline \multirow{2}{*}{$\begin{array}{l}\text { Institución educativa } \\
\text { Gustavo Rojas Pinilla }\end{array}$ 22 estudiantes } & Subcategoría & Estudiantes & Estudiantes \\
\cline { 2 - 4 } & Establece plan de solución. & 2 & 22 \\
\cline { 2 - 4 } & Comprueba los pasos ya realizados. & 1 & 18 \\
\cline { 2 - 4 } & Explica lo que hace y para qué lo hace. & 2 & 16 \\
\cline { 2 - 4 } & Soluciona el problema completamente. & 4 & 20 \\
\cline { 2 - 4 } & Reordena las ideas y prueba de nuevo. & 0 & 15 \\
\hline
\end{tabular}




\begin{tabular}{llcc}
\hline PROMEDIO & \multicolumn{2}{c}{19} \\
\hline \multicolumn{1}{l}{ DESVIACIÓN ESTÁNDAR } & 1 & 2,5 \\
\hline Concha Medina de & Estrablece plan de solución. & 1 & 9 \\
\cline { 2 - 4 } \begin{tabular}{l} 
9 estudiantes \\
\cline { 2 - 4 }
\end{tabular} & Comprueba los pasos ya realizados. & 1 & 6 \\
\cline { 2 - 4 } & Explica lo que hace y para qué lo hace. & 1 & 7 \\
\cline { 2 - 4 } & Soluciona el problema completamente. & 2 & 7 \\
\cline { 2 - 4 } & Reordena las ideas y prueba de nuevo. & 0 & 8 \\
\hline PROMEDIO & & 2 & 1,3 \\
\hline DESVIACIÓN ESTÁNDAR & 1,4 & 7 \\
\hline
\end{tabular}

Fuente: elaboración propia.

En lo que concierne a los resultados de los estudiantes en la etapa de aplicación del plan (ver tabla 20), la relación comparativa de los resultados de las IE, es el siguiente: $8.2 / 11.1$ en el pretest, mientras que en el postest es de 82.7 /
84.48, con una diferencia negativa para los estudiantes de la IE GRP de -1,7; en la primera fase, estos mostraron mayor participación. Nuevamente, se observan unas subcategorías en las que no hubo participación de los estudiantes de las dos IE.

Tabla 21

Etapa de revisión, verificación y utilización de subcategorías

\begin{tabular}{|c|c|c|c|}
\hline \multicolumn{4}{|c|}{ Resultados de los estudiantes en la etapa de revisión y verificación } \\
\hline & & Pretest & Postest \\
\hline $\begin{array}{l}\text { Institución } \\
\text { educativa }\end{array}$ & Subcategoría & Estudiantes & Estudiantes \\
\hline \multirow{6}{*}{$\begin{array}{l}\text { Gustavo Rojas } \\
\text { Pinilla } \\
22 \text { estudiantes }\end{array}$} & Son correctos los pasos y operaciones. & 2 & 20 \\
\hline & Establece con precisión cuál fue el paso clave en la solución. & 3 & 15 \\
\hline & $\begin{array}{l}\text { Colabora a sus compañeros para desarrollen habilidades en la } \\
\text { solución de situaciones. }\end{array}$ & 2 & 15 \\
\hline & $\begin{array}{l}\text { Utiliza el resultado obtenido para formular y plantear nuevas } \\
\text { situaciones. }\end{array}$ & 0 & 10 \\
\hline & Se autoevalúa. & 2 & 22 \\
\hline & Lee nuevamente el enunciado y comprueba. & 2 & 20 \\
\hline \multicolumn{2}{|l|}{ PROMEDIO } & 1 & 17 \\
\hline \multicolumn{2}{|c|}{ DESVIACIÓN ESTÁNDAR } & 2 & 4 \\
\hline \multirow{6}{*}{$\begin{array}{l}\text { Concha Medina } \\
\text { de Silva } \\
9 \text { estudiantes }\end{array}$} & Son correctos los pasos y operaciones. & 1 & 9 \\
\hline & Establece con precisión cuál fue el paso clave en la solución. & 2 & 7 \\
\hline & $\begin{array}{l}\text { Colabora a sus compañeros para que desarrollen habilidades en la } \\
\text { solución de situaciones. }\end{array}$ & 1 & 8 \\
\hline & $\begin{array}{l}\text { Utiliza el resultado obtenido para formular y plantear nuevas } \\
\text { situaciones. }\end{array}$ & 0 & 6 \\
\hline & Se autoevalúa. & 2 & 8 \\
\hline & Lee nuevamente el enunciado y comprueba. & 1 & 7 \\
\hline \multicolumn{2}{|l|}{ PROMEDIO } & 1 & 7 \\
\hline \multicolumn{2}{|c|}{ DESVIACIÓN ESTÁNDAR } & 0,4 & 1,4 \\
\hline
\end{tabular}

Fuente: elaboración propia. 
De la misma manera, en la etapa de revisión y verificación, los resultados porcentuales obtenidos respectivamente en las dos IE (ver tabla 21), son los siguientes: 8.3 / 13.0 y 77.3 / 83.3, observándose una diferencia porcentual positiva de 6,0 puntos a favor de la IE CMS, porque la relación de participación estudiantil es de $17(77.3 \%)$ y de 8 (88.9\%), lo cual se justifica en la notoria disminución en la subcategoría: utiliza el resultado obtenido para formular y plantear nuevas situaciones (10 estudiantes, 45.5 $\%)$.

\section{De la fase propositiva y de creación propia de los estudiantes}

Como una actividad de complementación y con el fin de identificar cómo el estudiante razona, comunica, modela, argumenta, propone, crea y resuelve problemas de aplicación matemática y se apropia de la estrategia implementada respecto a los pasos fundamentales expresados por Pólya en las estrategias para la solución de problemas; se les entregó a cada uno una hoja de papel tamaño oficio en blanco, cuyo propósito era que crearan un problema con las condiciones de su entorno y le dieran solución utilizando las subcategorías de cada etapa trabajada durante la investigación de aula y desarrollada en las instituciones GRP y CMS.

Cabe agregar que, la creatividad de los estudiantes fue desbordada, elaborando por decirlo así, composiciones matemáticas con sólidos despliegues de argumentación, llenas de habilidades, destrezas, prácticas y acciones, acompañadas del ingenio individual para expresar y adornar cada obra, demostrando con ello, que se atrajo, captó y retuvo su atención para enriquecer su aprendizaje en temas matemáticos, en este caso en la solución de problemas matemáticos y su aplicación a la realidad, al que con regularidad se le tiene aversión por la forma tradicional como se enseña.

Los estudiantes presentaron problemas referentes a temas como fraccionarios, enteros, medidas de superficie, de longitud, sistema decimal múltiplos y submúltiplos, destacando la presencia de situaciones de aplicación matemática con un nivel catalogado como medio, que involucró la realización de múltiples operaciones (suma, resta, división y multiplicación).

De la misma manera, la mayoría de los estudiantes construyó dos problemas, que incluyeron no solo las actividades que comúnmente se ven en los hogares y en los sitios de trabajo, especialmente en el campo, sino aquellas complementarias, que especialmente las madres y padres realizan cotidianamente, para generar sus ingresos, tales como: venta de comidas (tipo y valor); tienda (productos, que más se venden); prestación de servicio de acarreo; elaboración de queso y su comercialización (precio por unidad y su presentación); cultivo de papa, con su comercialización (tienen en cuenta las variedades, precio de venta del bulto de cuatro arrobas); el tamaño del área de la finca, precio de la fanegada según la calidad de la tierra; panadería
Los estudiantes presentaron problemas referentes a temas como fraccionarios, enteros, medidas de superficie, de longitud, sistema decimal múltiplos y submúltiplos, destacando la presencia de situaciones de aplicación matemática 
(productos más vendidos y precio); miscelánea (actividades principales, con productos más destacados y precio, incluyendo útiles escolares); engorde y venta de cerdos (precio en pie); cría de gallinas y su comercialización (precio de venta unitario según temporada), cría de ganado vacuno, incluye comercialización (precio de venta en pie, con cría o sin ella); comercialización de la leche que obtienen de las vacas paridas (precio de venta, según su presentación).

Las descritas anteriormente, son situaciones cotidianas que viven a diario los estudiantes, junto con los demás miembros de la familia, y de ella aprendieron las diferentes circunstancias que se derivan de cada una, al igual que los participantes y el rol que desempeña cada individuo, las cuales les sirvieron como argumento para recrear su producción matemática, rodeadas de categorías conceptuales a través de las cuales, enriquecieron el proceso de enseñanza aprendizaje en este tema. Son acciones donde la duda $\mathrm{y}$ el temor, mostrados en el pretest $\mathrm{y}$ parte del postest, fueron superadas, además de evidencia de la relación entre el aprendizaje y la práctica social (Riscanevo \& Jiménez, 2017).

En cuanto a las competencias, estas se construyeron, desarrollaron y evolucionaron, más allá de las perspectivas que se tenían. Los estudiantes utilizaron, con mayor énfasis, la resolución de problemas, luego las comunicativas $\mathrm{y}$ hasta el razonamiento.

Respecto al aprendizaje, los estudiantes demostraron apropiación de los conocimientos compartidos impartidos, además de habilidades y capacidades que superaron las expectativas, frente a las acciones pedagógicas propuestas, especialmente, al construir ellos mismos una situación o problema, el cual resolvieron con lujo de detalles y argumentos.

Así mismo, en las diferentes evidencias, se observa y comprueba el desempeño de los estudiantes, el cual estuvo rodeado de alegría y creatividad, a través de secuencias explicativas apoyadas en modelización matemática, donde hacen presencia diversos métodos para llegar a la respuesta, pero sobre todo este ejercicio permitió a cada alumno apropiarse y enriquecerse de algo que ya podía saber, pero que no había explorado y que le permite ser aplicado para problemas que ha de enfrentar en otras asignaturas y en su vida.

Cabe destacar, el caso de un estudiante de la IE Gustavo Rojas Pinilla quien en la prueba pretest manifestó por escrito no entender nada, según su maestra presenta presuntamente cierta dificultad y apatía por las matemáticas, siempre se muestra distraído y su ritmo de aprendizaje en matemáticas es pausado con respecto a sus compañeros, manifiesta que su situación familiar desde siempre ha sido complicada. En esta actividad, él elaboró dos problemas, uno relacionado con cultivo de papa (venta en bultos) y la segunda venta de leche (litros) y deducciones (alimentación, compra de otros insumos). En la etapa de comprensión, presentada por este estudiante, a través de dibujos de cada uno de los personajes y elementos utilizados para su situación 
problema, se deduce que el niño vive con el personaje de mayor importancia (el abuelo, a quien muestra muy elegante); asimismo, evidencia en su dibujo cada bulto de papa con su precio de venta, la cantidad y el tipo (gruesa, tercera y riche, que es para el sustento), además, con toda esta descripción están los datos que debe tener y que se piden en un problema, así como las incógnitas pertinentes encontrando una buena relación entre estos.

Respecto a la etapa de planeación, determina todos los datos con sus características (cantidades y precio de venta), con las respectivas condiciones y se apoya el método utilizado anteriormente. La etapa de desarrollo del plan contiene la solución a través de varias operaciones matemáticas planteadas, comprueba los pasos, explica qué hace y por qué lo hace, soluciona el problema con las ideas y verifica los resultados.

La etapa de valoración y verificación, relaciona las diversas respuestas obtenidas, determinando los pasos claves en la solución, se autoevalúa, lee los resultados y los verifica. Por otra parte, en cuanto al desarrollo de las diversas situaciones planteadas y resueltas por los estudiantes, a nivel general, en las dos IE se observa lo siguiente en las diferentes etapas:

Etapa de comprensión. Contiene dibujos de personas, animales, productos agrícolas y cosas, con sus características apoyadas con colores; esquemas explicativos de los diferentes procesos que se llevan a cabo; árbol de problemas y figuras geométricas, que dan a entender lo que significa el problema, con los datos que aporta y los que se deben hallar, reconociendo que existen similitudes con otros que ya se han resuelto en clase.

Etapa de planeación. Ubica los datos, que los asocia con las estrategias y condiciones a utilizar, a través del razonamiento directo, determinando las diversas operaciones que debe realizar (con la justificación individual de cada aspecto a determinar) secuencialmente para llegar a las respuestas.

Etapa de desarrollo del plan. Con las estrategias seleccionadas para solucionar el problema, plantea y desarrolla cada una de las operaciones que permiten responder los interrogantes planteados en la etapa anterior.

Etapa de valoración y verificación. En este caso, al mirar hacia atrás, se verifican las respuestas obtenidas a través de la comprobación. Se propende por la búsqueda de otra respuesta más sencilla, y se determina la posible aplicación de esta solución a un caso general, de otra asignatura o de la vida real.

Al retomar los resultados obtenidos de las cinco situaciones expuestas y resueltas por los estudiantes de las dos instituciones educativas, mediante el pretest, el postest, la aplicación de talleres con el apoyo de los docentes y la última intervención voluntaria de los primeros; se concluye que el docente, en su mayoría, pretende que el alumno se amolde a las condiciones de la IE en las enseñanzas de las matemáticas y no que esta haga lo propio con el apoyo de los docentes, para facilitar ese proceso de 
A manera de conclusión, las situaciones descritas llevan a distintos planteamientos frente a la posibilidad que se tiene que cambiar la actitud de los docentes y la manera de llevar a los estudiantes situaciones problema, que se han venido aplicando. enseñanza aprendizaje, que como en el caso del apoyo de Pólya en Matemáticas, los motiva, despierta su creatividad y atrae la atención para generar ese cambio que se debe generar sin tanto esfuerzo.

Es por ello que, el docente, además de interesarse por su materia y la conoce, debe -como se dijo anteriormente- ponerse en los zapatos de los estudiantes, utilizando estrategias y medios que permiten explorar sus dificultades, expectativas y potencialidades, para aprovecharlas y mejorar su rendimiento académico, lo que se logra a través de la promoción de actitudes mentales y creando hábitos de trabajo metódico, disponiéndose para que conjeturen, comprueben, verifiquen $\mathrm{y}$, en cierta forma, jueguen con sus expectativas $\mathrm{y}$, sobre todo, asocien lo analizado con problemas comunes que se presentan al individuo, la familia, la escuela o la sociedad. Hay que promover la crítica constructiva argumentada y sustentada en elementos sólidos.

A manera de conclusión, las situaciones descritas llevan a distintos planteamientos frente a la posibilidad que se tiene que cambiar la actitud de los docentes y la manera de llevar a los estudiantes situaciones problema, que se han venido aplicando. Un primer paso necesario para la implementación de cambios significativos, podría centrarse en el análisis crítico para ir descubriendo, de una manera más fácil y viable, al abordar un problema, la importancia de poderlos resolver de una manera adecuada.

Con la aplicación de la propuesta pedagógica, se genera un ambiente propicio, atrayente, que capta y cautiva la atención de los estudiantes, máxime cuando se apoya en material didáctico fácil de elaborar, acorde con la edad de los estudiantes y el plan de estudios.

Se comprueba que al resolver una situación problema siguiendo unas etapas, se facilita el aprendizaje y se mejora el nivel de logro en los estudiantes despertando el interés por resolver las situaciones problema de manera diferente, manifestando el gusto por resolver situaciones de aplicación matemática para poder superar sus dificultades.

Por otra parte, es relevante reconocer que con la aplicación del método de Pólya, no solo se genera un cambio en los estudiantes con quiénes se aplica, sino también en los docentes que intervinieron en ella, porque enseña que son múltiples las alternativas y la riqueza existente en el medio que pueden aprovecharse y contribuir significativamente en el proceso de enseñanza del alumno y del propio, fortaleciendo las competencias no solo en esta área, sino para enfrentar situaciones en la vida práctica y darle soluciones.

Se observa y comprueba el desempeño de los estudiantes, el cual estuvo rodeado de alegría y creatividad, a través de secuencias explicativas apoyadas en modelización matemática, donde hacen presencia diversas estrategias para abordar una situación problema de aplicación matemática, pero sobre todo este ejercicio permitió a cada estudiante apropiarse y enriquecer algo que ya podía saber, pero que no había explorado y que le permite ser aplicado para problemas que ha de enfrentar en otras asignaturas $\mathrm{y}$ en vida cotidiana. 


\section{Referencias}

Agudelo, G., Bedoya, V., \& Restrepo, A. (2008). Método beurístico en la resolución de problemas matemáticos. Pereira: Universidad Tecnológica de Pereira.

Barajas, P. (2013). Estrategias de aprendizaje basadas en la resolución de problemas. Tunja: Universidad Pedagógica y Tecnológica de Colombia.

Blanco, L. (1991). Conocimiento y acción en la enseñanza las Matemáticas, de profesores de E.G.B. y estudiantes para profesores. Universidad de Extremadura. Cáceres.

Calvo, M. (2007). Enseñanza eficaz de la resolución de problemas en matemáticas. San José: Universidad de Costa Rica.

Cortés, M., \& Galindo, N. (2006). El modelo de Pólya centrado en resolución de problemas en la interpretación y manejo de la integral definida. (Tesis de grado). Universidad de La Salle, Bogotá, Colombia.

Elliot, J. (2000). La investigación acción en educación. Madrid: Morata.

Escalante, S. (2015). Método Pólya en la resolución de problemas matemáticos. Municipio de La Democracia, departamento de Huehuetenango (Guatemala): Escuela Oficial Rural Mixta "Bruno Emilio Villatoro López”.

Fernández, J. (2006). Algo sobre resolución de problemas matemáticos en educación básica primaria. Sigma, (29), 29-42.

Hernández, R., \& Fernández, C. (1998). Metodología de la investigación. México: McGraw-Hill.

Kemmis, S., \& McTaggart, R. (1988). Cómo planificar la investigación-acción. Barcelona: Laerles.

Llivina, M.J. (1999). Una propuesta metodológica para contribuir al desarrollo de la capacidad para resolver

problemas matemáticos. Tesis de Doctorado, La Habana.

Murillo, M. (2013). Prácticas de enseñanza empleadas por docentes de matemáticas y su relación con la resolución de problemas. (Artículo para congreso). Universidad de Antioquia.

Pólya, G. (1984). Cómo plantear y resolver problemas. México: Trillas.

Riscanevo, L. E., \& Jiménez, A. (2017). La experiencia y el aprendizaje del profesor de matemáticas desde la perspectiva de la práctica social. Praxis \& Saber, 8(18), 203-232. https://doi.org/10.19053/22160159.v8.n18.2017.7249 
Romero, S. (2011). La resolución de problemas como herramienta para la modelización matemática. España: Universidad de Huelva.

Villagrán, M., \& Navarro, J. (2000). Aplicación de una estrategia de resolución de problemas matemáticos en niños. (Tesis de grado). Universidad de Cádiz, Cádiz, España. 